,

\title{
IUVDT-Second African Regional Conference on sexually transmitted diseases
}

\author{
Report of the conference on STD held by the African Branch of the International Union against the \\ Venereal Diseases and Treponematoses in Dakar, Senegal, 4-9 May 1981
}

The Second African Regional Conference on sexually transmitted diseases of the African Branch of the International Union against the Venereal Diseases and Treponematoses was held in Dakar, Senegal, from 4 to 9 May 1981. Seventyeight members and invited guests took part, representing 24 different countries.

The opening ceremony took place in the presence of the secretary of state for scientific and technical research and the dean of the medical school. The International Union against the Venereal Diseases and Treponematoses (IUVDT) was represented by the president, Dr R D Catterall, and the World Health Organisation by Dr G M Antal of Geneva.

\section{Scientific sessions}

- The organising committee headed by Professor I Diop Mar and the general secretary, Associate Professor B Ndiaye, had prepared an extensive programme covering the whole spectrum of sexually transmitted diseases. The first morning was devoted to general epidemiology, and the development of STD in Africa was reviewed by Dr A Retel-Laurentin (France). The service of STD control in Nigeria was described by Dr Sanu Alli, and some observations on the situation in Senegal were presented by Dr Ndoye.

\section{GONORR HOEA}

The session on gonorrhoea which followed included a description of the discovery of a new strain of B-lactamase-producing gonococcus detected in Rotterdam by Professor E Stolz (Holland) and of the spread of penicillinase-producing strains of Neisseria gonorrhoeae (PPNG) throughout central Africa by Professor A O Osoba (Nigeria). Dr Stuart Brown (USA) said that there had been a $400 \%$ increase in the number of reported PPNG strains between 1979 and 1980 in the USA, and $21 \%$ of women with PPNG strains had salpingitis. Evidence from Los Angeles recently suggested that a major epidemic of PPNG strains might be expected in California and especially in Los Angeles itself. Professor M W Adler (London) described the trends for gonorrhoea, pelvic inflammatory disease, and PPNG in England. There had been a marked increase in reported PPNG strains in the United Kingdom and a marked rise in reported cases of pelvic inflammatory disease during the past three years. Confirmation that PPNG strains were circulating in Senegal came from $\mathrm{Dr}$ Buisson and his colleagues from the Institut Pasteur in Dakar. The world situation of the PPNG strains was reviewed by Dr G M Antal of the World Health Organisation, who pointed out that these strains were increasing in all the countries of the world from which reports were being received. Formerly, constant antibiotic pressure from the misuse of antibiotics seemed necessary for the development of PPNG strains, but this was probably no longer the case and all the evidence points to a further rapid increase in resistant strains throughout the world.

A number of papers describing trials of various antibiotics in the treatment of gonorrhoea-including thiamphenicol, cefoxitin, amoxycillin, and spectinomycin-were read. Professor A Z Meheus (Belgium) described the sensitivity of several African strains of $N$ gonorrhoeae and pointed out that the dilution technique was much more reliable than the disc method in assessing sensitivity. Even in the presence of chromosomal resistance treatment with procaine penicillin and probenecid was still valid, but for PPNG strains spectinomycin or another appropriate antibiotic was required; this conclusion was confirmed by the work of Dr $\mathbf{R}$ Widy-Wirsk in Central Africa. The prophylaxis of gonococcal conjunctivitis of the newborn, first described by Crede 100 years ago, was reviewed by $\mathrm{Dr} \mathrm{D} \mathrm{H} \mathrm{H}$ Robertson (Edinburgh).

\section{SYPHILIS}

The incidence of syphilis in Egypt, Senegal, and other African countries was described and the falling incidence of congenital,,$\vec{\omega}$ cardiovascular, and neurosyphilis in Dakar $\stackrel{\text { ? }}{=}$. outlined. Cerebral vascular lesions andér general paralysis were the commonest. forms of neurosyphilis seen in Senegal, and tabes dorsalis was uncommon.

\section{GENITAL ULCERS}

The highest standards of both the papersand the discussion which followed were reached in the session on genital ulceration in Africa. Professor $\mathbf{H}$ Nsanze (Kenya) described the diagnosis and treatment of aD series of patients seen in Nairobi and Professor A Z Meheus (Be'gium) reported the preliminary results of a long-term study on genital ulceration being undertaken in Swaziland. Chancroid, syphilis, ando lymphogranuloma venereum were thes commonest diagnoses made, but in both series no definite diagnosis was made in over $20 \%$ of cases. It is of interest to thosed from Europe and North America that herpes simplex was diagnosed in less thano $10 \%$ of cases in both series.

PELVIC INFLAMMATORY DISEASE Pelvic inflammatory disease (PID) was said to be responsible for $6 \%$ of alf gynaecological admissions to hospital in France by $\mathrm{Dr}$ A Siboulet (Paris). The aetiology of the condition had changed recently, and sexually transmitted diseases had replaced abortion as the commonesto cause. Three more commonly recognised causes were chlamydia, ureaplasmas, ant mycoplasmas, but the best microbiologicaE. results were obtained when the laboratory was brought into the operating theatre and specimens were taken and inoculated during laparotomy or laparoscopy. Tubatu samples, brushing of the tubes and fimbriae with special brushes, and microbiopsies helped to establish the diagnosis but in $50 \%$ of sterile women with salpingitis no cause could still be found for the tubal damage.

OTHER TOPICS

Other subjects on which papers were presented included group B streptococci, Gardnerella vaginalis, Haemophilus 
ducreyi, Trichomonas vaginalis, and hepatitis B. The scientific part of the conference ended with the presentation of a modern stereo multiple-image tape and slide film by Dr A Siboulet (Paris), which had been prepared for educational purposes.

\section{General business}

The general assembly of the African Branch of the IUVDT re-elected Professor I Diop
Mar as president and Professor A O Osoba as general secretary. All the other officers were also re-elected for a further period of two years. The statutes and bye-laws of the branch have not yet been completely translated into French and await confirmation by the general assembly when this is completed.

It was unanimously agreed that the next meeting should be in Nairobi, Kenya, in 1983. The exact date is to be decided later.
Most of the delegates stayed at the Taranga Hotel, Dakar, and there was an excellent social programme, which include a cocktail party, a dinner, a buffet suppet, and a variety of excursions in the region

There was general agreement that African Branch of the IUVDT was nespiv fully launched on its course and that gs second international meeting had been $\mathrm{g}^{n}$ unqualified success.

\section{Notice \\ CHANGE OF EDITOR}

As from 1 January 1982, Dr A McMillan will be Editor of the BJVD. All papers for possible publication should be sent to: Dr A McMillan, Wards 45 and 46, Royal Infirmary, Edinburgh EH3 9YW, as from that date. 